\title{
Bronchopulmonary dysplasia: incidence and severity in premature infants born at high altitude.
}

\author{
Jefferson Buendia ${ }^{1}$, Cristian Ramieez ${ }^{2}$, and Dione Benjumea ${ }^{2}$ \\ ${ }^{1}$ University of Antioquia \\ ${ }^{2}$ UNIREMINGTON Facultad de Ciencias de la Salud
}

September 25, 2021

\begin{abstract}
Background: Bronchopulmonary dysplasia (BPD) is the most common cause of chronic lung disease in children born prematurely. There is little information about the epidemiology and severity of BPD places with high altitude. This study aimed to evaluate the frequency of BPD severity levels and the associated risk factors with severity in a cohort of preterm newborns [?]34 weeks of gestational age born in Rionegro, Colombia Materials and methods: We carried out a retrospective analytical cohort of preterm newborns without major malformations from Rionegro, Colombia between 2011-2018 admitted to neonatal intensive unit at high altitude $(2200 \mathrm{~m}$ above sea level). The main outcomes were the incidence and severity of bronchopulmonary dysplasia. Results: The bronchopulmonary dysplasia incidence was 25.7\% (95\% CI, 21.6-29.9). Bronchopulmonary dysplasia was moderate in $62.1 \%$ of patients and severe in $26.7 \%$. After modeling regression analysis, the final variables associated with BPD severity levels were: sepsis (OR 2.37 CI 95\% 1.04-5.40) and pulmonary hypertension (OR 3.79 CI95\% 1.19-12). Conclusion: The incidence of BPD was higher and similar to cities with higher altitudes. In our population, the variables associated with BPD severity levels were: duration of oxygen therapy and pulmonary hypertension. It is necessary to increase the awareness of risk factors, the effect of clinical practices, and early recognition of bronchopulmonary dysplasia to reduce morbidity in patients with this pathology.
\end{abstract}

\section{Bronchopulmonary dysplasia: incidence and severity in premature infants born at high alti- tude.}

Jefferson Antonio Buendía ${ }^{1}$, Cristian Ramírez ${ }^{2}$ Dione Benjumea-Bedoya ${ }^{2}$,

1 Grupo de Investigación en Farmacología y Toxicología. Departamento de farmacología, Facultad de Medicina, Universidad de Antioquia

2. Corporación Universitaria Remington

Corresponding author: Jefferson A. Buendía, MD, PhD, Facultad de Medicina, Universidad de Antioquia, Carrera 51D \#62-29, Tel: (+574) 2196046; Medellín, Colombia. E-mail: jefferson.buendia@gmail.com. E-mail: JAB:jefferson.buendia@gmail.com

Statement of financial support: This work was carried out with the authors' own funds.

Disclosure statement: No conflict of interest

\section{Abstract}

Background: Bronchopulmonary dysplasia (BPD) is the most common cause of chronic lung disease in children born prematurely. There is little information about the epidemiology and severity of BPD places with high altitude. This study aimed to evaluate the frequency of BPD severity levels and the associated 
risk factors with severity in a cohort of preterm newborns [?]34 weeks of gestational age born in Rionegro, Colombia

Materials and methods: We carried out a retrospective analytical cohort of preterm newborns without major malformations from Rionegro, Colombia between 2011-2018 admitted to neonatal intensive unit at high altitude (2200m above sea level). The main outcomes were the incidence and severity of bronchopulmonary dysplasia.

Results: The bronchopulmonary dysplasia incidence was $25.7 \%$ (95\% CI, 21.6-29.9). Bronchopulmonary dysplasia was moderate in $62.1 \%$ of patients and severe in $26.7 \%$. After modeling regression analysis, the final variables associated with BPD severity levels were: sepsis (OR 2.37 CI 95\% 1.04-5.40) and pulmonary hypertension (OR 3.79 CI95\% 1.19-12).

Conclusion: The incidence of BPD was higher and similar to cities with higher altitudes. In our population, the variables associated with BPD severity levels were: duration of oxygen therapy and pulmonary hypertension. It is necessary to increase the awareness of risk factors, the effect of clinical practices, and early recognition of bronchopulmonary dysplasia to reduce morbidity in patients with this pathology.

Keywords: Bronchopulmonary dysplasia, severity, incidence, Colombia, oxygen, hypertension

\section{Abbreviations}

Bronchopulmonary dysplasia (BPD)

Neonatal intensive critical unit (NICU)

Pulmonary arterial hypertension $(\mathrm{PH})$

\section{Introduction}

Bronchopulmonary dysplasia (BPD) is the most common cause of chronic lung disease in children born prematurely(1). This condition has an increasing incidence, mainly in low- and middle-income countries, generating higher rates of respiratory symptoms at follow-up, and increased healthcare utilization(2). Previous epidemiological studies have identified prematurity, oxygen toxicity, excess fluid administration, and mechanical ventilation as major risk factors associated with $\operatorname{BPD}(3)$. Recent advances in life support, including the use of antenatal steroids and early management with a surfactant, increased survival in lower gestational ages, but it also increased the incidence of $\mathrm{BPD}(4)$.

There is little information about the epidemiology and severity of BPD at high altitude especially in developing countries. The knowledge of the severity has cardinal importance in the planning of patient follow-up, given to BPD severity levels demonstrate an adequate correlation with structural lung changes and respiratory morbidity after neonatal intensive critical unit (NICU) discharge (4). Knowing a priori the variables associated with disease severity allows stratifying care according to risk, thus ensuring more timely care with better patient outcomes (2-4) This is even more relevant in high altitude locations where there is less evidence and where altitude may play a key factor in the pathogenesis of the disease. We conducted a study aimed to evaluate the frequency of BPD severity levels and the associated risk factors with severity in a cohort of preterm newborns without major malformations from Rionegro, Colombia.

\section{Materials and methods:}

Study population.

An observational, retrospective cohort study was designed. Medical records of all of preterm newborns ( gestational [?]36 weeks postmenstrual age ) who were born at Clinica Somer, Rionegro from January 2011 and January 2018 were reviewed $(\mathrm{n}=451)$. Rionegro is a city at 2200 meters above sea level in the northeast of Colombia. We included patients who meet the definition of BDP according to the National Heart, Lung, and Blood Institute (NHLBI) (5). Using this definition bronchopulmonary dysplasia is present when supplemental oxygen at any concentration for 28 days in premature newborns with gestational age 
[?]32 weeks at birth is needed. All research was performed in accordance with relevant guidelines/regulations approved by the Institutional Review Board of Clinica Somer and Uniremington University.

Definition of BPD and respiratory outcomes.

BPD severity was defined according to the National Heart, Lung, and Blood Institute (NHLBI) (5). Severity was also defined based on oxygen requirements at 36 weeks postmenstrual age or at discharge in the following manner: If no supplemental oxygen was required it was classified as mild, if oxygen requirements were less than $0.3 \mathrm{FiO} 2$ it was considered as moderate, and if the requirements were greater than $0.3 \mathrm{FiO} 2$ or if there was a need of some type of ventilator support (invasive, noninvasive), it was considered severe $(5,6)$. Oxygen requirement is defined as when the patient presents saturation values for hemoglobin pulse oximetry below $90 \%$ at sea level.

At baseline, we collected information on the number of courses of antenatal corticosteroids for the acceleration of fetal lung maturation, gender, birth weight, duration of neonatal ventilatory support, maternal age, apgar score, maternal comorbidities, and complications such as pulmonary arterial hypertension $(\mathrm{PH})$ (increased pulmonary vascular resistance and presence of right -left shunt at the foramen ovale and or ductus arteriosus level. Echocardiography was used as screening modality for $\mathrm{PH}$ using tricuspid regurgitant jet to estimate systolic pulmonary artery pressure) (7), persistent ductus arteriosus, neonatal pneumonia, hyaline membrane disease, sepsis, chorioamnionitis (positive amniotic fluid test result (gram stain, glucose level, or culture results consistent with infection) or placental pathology demonstrating histologic evidence of placental infection or inflammation) . Neonatologist utilized oximetry to assess for true oxygen dependence; and there a correction was applied for altitude (10). Oxygen requirement is defined as when the patient presents saturation values for hemoglobin pulse oximetry ( $\mathrm{SpO} 2$ ) below $90 \%$ at sea level. For the altitude adjustment, we use the same procedure described by Velasquez (8), Briefly we took into account the FiO2 at which an inspiratory oxygen pressure is similar to that found at sea level with a FiO2 of 0.21 . In order to classify the severity, the same equation was used, but utilizing a FiO2 of 0.3 that resulted in an equivalent $\mathrm{FiO} 2$ of 0.41 . With these calculations, the BPD classification adjusted for Rionegro was defined as the use of supplemental oxygen with a FiO2 of at least 0.29 for 28 days. For the severity classification, it was defined as mild if patient had no supplemental oxygen by week 36 PMA or discharge, moderate if patients had oxygen requirement of $0.29-0.41$ by 36 weeks PMA or discharge, and it was defined as severe if the oxygen requirements were greater than 0.42 or if they required some kind of assisted ventilation. To determine the $\mathrm{FiO} 2$ in patients using conventional cannulas we use the approximation of Benaron (tidal volume [TV] of 6 $\mathrm{ml} / \mathrm{kg}$ and an inspiratory time (IT) of $0.33 \mathrm{~s}$ ) (8)

All patients were treated following the recommendation about prevention, diagnosis, resuscitation, early management and treatment of bronchopulmonary dysplasia established in the national guide for the management of the premature newborn $(10,11)$

\section{Statistical analysis.}

Descriptive statistics are presented as mean and standard deviation. Group comparisons were performed using t-test, Mann-Whitney U-test, and chi2-test, as appropriate. In order to identify factors independently associated with BPD severity levels, we fit the data to partial proportional odds models for ordinal dependent variables (ordinal logistic regression). Candidate confounders were selected based on a review of the literature and biological plausibility. Regression results are reported as odds ratios and their respective $95 \%$ confidence intervals (CI). All statistical tests were two-tailed, and the significance level used was $\mathrm{p}<0.05$. The data were analyzed with the Statistical Package Stata 12.0 (Stata Corporation, College Station, TX, USA).

\section{Results.}

Baseline characteristics of preterm newborns by gestational age are presented in Table 1. As expected, statistically significant differences by gestational age were found in variables such as birth weight, duration of supplemental oxygen, oxygen use at discharge, use of surfactant therapy and antenatal corticosteroids, frequency of $\mathrm{BPD}$, hyaline membrane disease and patent ductus arteriosus. 
116 met the criteria for bronchopulmonary dysplasia ( incidence of $25.7 \% 95 \%$ CI, 21.6-29.9). Bronchopulmonary dysplasia was mild in $11 \%$, moderate in $62 \%$ and severe in $27 \%$ of patients. Among patients with different levels of BPD severity, there were statistically significant differences in birth weight, duration of supplemental oxygen, oxygen use at discharge, frequency of sepsis and pulmonary hypertension, see table 2. After modeling regression analysis, the final variables associated with BPD severity levels were: sepsis (OR 2.37 CI $95 \%$ 1.04-5.40) and pulmonary hypertension (OR 3.79 CI95\% 1.19-12), see table 3.

\section{Discussion.}

In this study population, the incidence of BPD was $25.7 \%$. Our incidence of BPD is higher than rates reported in developed countries but similar to cities with higher altitudes in Colombia such as Bogota(12,13). A previous case-control study found variations in the prevalence of BPD according to altitude in Colombia. In cities located a higher altitude (more than 2600 meters above sea level), the prevalence of BPD were higher than other cities with lower altitude as Bucaramanga and Cali (10). Highest altitude city (Bogota) was associated with a higher risk of dysplasia (OR 1.82 95\% CI 1.31-2.53) (12). Infants who are born in cities with higher altitudes ( $>2000$ meters above sea level) have twice the risk of developing BPD than infants born in cities with lower altitudes, independently of differences in maternal, infant, and therapeutic risk factors $(12,14)$. Altitude play an important role in the pathogenesis of BPD. The decreased partial pressure of oxygen in the environment, in cities with higher altitudes, has also been associated with higher pulmonary artery pressures, delayed in the functional closure of the ductus arteriosus in the newborn, tortuous transition from oxygenation via placenta to oxygenation across the lungs resulting in postnatal persistence of fetal circulation(15). As a result of these events, it is possible that premature infants born at high altitudes have an early dependency on high concentrations of oxygen and ventilatory support compared to patients born at lower altitudes. In our study, sepsis was a risk factor associated with BPD severity. Multiple studies indicate that postnatal sepsis independently increases the incidence of BPD

Preterm infants are more susceptible to infections since their immune defenses are not fully developed, have vulnerable skin barrier, and require multiple invasive procedures. Late-onset sepsis induces a proinflammatory and pro-fibrotic response in the preterm lung predisposing it to BPD . Neonatal mice injected with intraperitoneal LPS demonstrated reduced lung inflammation and apoptosis after $24 \mathrm{~h}$ as compared to adults, and this was associated with activation of the transcription factor, nuclear factor kappa $\mathrm{B}$. Inhibition of NF- $\chi \mathrm{B}$ resulted in increased cell death and alveolar simplification and disruption of angiogenesis via vascular growth factor (VEGF)-R2 (9-11)

We found that pulmonary arterial hypertension $(\mathrm{PH})$ was an independent predictor of $\mathrm{BPD}$ severity. $\mathrm{PH}$ is a common complication of neonatal respiratory diseases including BPD. Up to $18 \%$ of all extremely low birth weight infants will develop some degree of $\mathrm{PH}$, and the incidence rises to $25-40 \%$ of infants with established BPD (12). PH worsens the clinical course, morbidity, and mortality of $\mathrm{BPD}(12)$. PH also is a common disease in individuals living at high altitudes, yet this is a understudied disorder especially in patients with BPD (13). A better understanding of the pathogenesis is necessary to optimize current drugs and newer therapeutic targets.

A number of important limitations need to be considered. As occurs in longitudinal studies, information bias cannot be excluded. However we include all patients registered in the hospital and the reported incidence cannot be attributed to the bias of selection; nor can it be attributed to overdiagnosis of oxygen dependence, since the neonatologist in all patient verified with physiological tests (dynamic oximetry standardized) that children truly require supplemental oxygen. Second, hospitalizations due to other medical conditions sometimes can prolong the hospital stay and the withdrawal of oxygen (inefficiencies within the healthcare system, placement difficulties, operational delays, and payer-related issues). However, the plausibility of the identified clinical factors suggests that the above-mentioned non-medical factors account for a minimal part of the causality in the studied population. Third, in our study used the echocardiography for PH diagnosis. However in infants with BPD can be especially challenging due to the presence of lung hyperinflation and heart rotation, both of which impact the sensitivity of imaging and identification of the tricuspid regurgitant jet. The echocardiography correctly diagnosed the presence or absence of PH $79 \%$ of the time, but correctly 
determined the severity PH only $47 \%$ of the time. These results imply that in the absence of a quantifiable TR jet, the currently available indirect measurements are insufficient for the assessment of the degree of $\mathrm{PH}$ in infants with $\operatorname{BPD}(13)$.

In conclusion, the incidence of BPD was higher than the average rates reported in populations with similar gestational ages in developed countries but similar to cities with higher altitudes. In our population, the variables associated with BPD severity levels were: duration of oxygen therapy and pulmonary hypertension. It is necessary to increase the awareness of risk factors, the effect of clinical practices, and early recognition of bronchopulmonary dysplasia to reduce morbidity in patients with this pathology.

Acknowledgements: None

\section{References}

1. Ali Z, Schmidt P, Dodd J, Jeppesen DL. Bronchopulmonary dysplasia: a review. Arch Gynecol Obstet. 2013;288(2):325-33.

2. Ruiz-Pelaez JG, Charpak N. [Bronchopulmonary dysplasia epidemic: incidence and associated factors in a cohort of premature infants in Bogota, Colombia]. Biomedica. 2014;34(1):29-39.

3. Li Y, Wei QF, Pan XN, Meng DH, Wei W, Wu QP. [Influencing factors for severity of bronchopulmonary dysplasia in preterm infants]. Zhongguo Dang Dai Er Ke Za Zhi. 2014;16(10):1014-8.

4. Rojas MX, Rojas MA, Lozano JM, Rondon MA, Charry LP. Regional variation on rates of bronchopulmonary dysplasia and associated risk factors. ISRN Pediatr. 2012;2012:685151.

5. Ehrenkranz RA, Walsh MC, Vohr BR, Jobe AH, Wright LL, Fanaroff AA, et al. Validation of the National Institutes of Health consensus definition of bronchopulmonary dysplasia. Pediatrics. 2005;116(6):1353-60.

6. Bhandari V. Designing a better definition of bronchopulmonary dysplasia. Pediatr Pulmonol. 2019;54(6):678-9.

7. Bui CB, Pang MA, Sehgal A, Theda C, Lao JC, Berger PJ, et al. Pulmonary hypertension associated with bronchopulmonary dysplasia in preterm infants. J Reprod Immunol. 2017;124:21-9.

8. Vasquez P, Romero Diaz HA, Gomez Gomez MJ, Leal L, Yejas Navarro LM. Bronchopulmonary dysplasia predictor scale validation in preterm newborns in two neonatal units at $2600 \mathrm{~m}$ above sea level. Infant Behav Dev. 2018;52:1-8.

9. Alvira CM, Abate A, Yang G, Dennery PA, Rabinovitch M. Nuclear factor-kappaB activation in neonatal mouse lung protects against lipopolysaccharide-induced inflammation. Am J Respir Crit Care Med. 2007;175(8):805-15.

10. Iosef C, Alastalo TP, Hou Y, Chen C, Adams ES, Lyu SC, et al. Inhibiting NF-kappaB in the developing lung disrupts angiogenesis and alveolarization. Am J Physiol Lung Cell Mol Physiol. 2012;302(10):L1023-36.

11. Balany J, Bhandari V. Understanding the Impact of Infection, Inflammation, and Their Persistence in the Pathogenesis of Bronchopulmonary Dysplasia. Front Med (Lausanne). 2015;2:90.

12. Valenzuela D, Moya F, Luco M, Tapia JL. [The role of pulmonary hypertension on bronchopulmonary dysplasia]. Rev Chil Pediatr. 2017;88(6):699-706.

13. Berkelhamer SK, Mestan KK, Steinhorn RH. Pulmonary hypertension in bronchopulmonary dysplasia. Semin Perinatol. 2013;37(2):124-31.

\section{Hosted file}

Table 1 new 140921.docx available at https://authorea.com/users/316889/articles/538942bronchopulmonary-dysplasia-incidence-and-severity-in-premature-infants-born-at-highaltitude 


\section{Hosted file}

Table 2.docx available at https://authorea.com/users/316889/articles/538942-bronchopulmonarydysplasia-incidence-and-severity-in-premature-infants-born-at-high-altitude

Hosted file

Table 3.docx available at https://authorea.com/users/316889/articles/538942-bronchopulmonarydysplasia-incidence-and-severity-in-premature-infants-born-at-high-altitude 